\title{
O consumo de bebida alcoólica e o binge drink entre os graduandos de Medicina de uma Universidade de Minas Gerais
}

The consumption of alcoholic beverages and the binge drink among Medicine graduating in a Minas Gerais University

Thalles Trindade de Abreu', Amanda de Oliveira Maurílio', Caio César Liguori', Daniel Vasconcelos de Pinho Tavares', Delmo Manoel Gomes Terceiro', Lucas G. Malta Cunha', Vinícius Silva Belo', Alexandre Ernesto Silva ${ }^{1}$

\section{RESUMO}

Objetivos: $\mathrm{O}$ estudo objetivou verificar o padrão de consumo de bebidas alcoólicas e a presença do beber pesado episódico (BPE) entre os estudantes de Medicina de uma universidade do centro-oeste de Minas Gerais. Métodos: Trata-se de um estudo transversal, exploratório e descritivo, realizado com 202 graduandos do curso de Medicina de uma universidade do centro-oeste de Minas Gerais. A coleta de dados foi realizada no primeiro semestre de 2015, com a utilização de um questionário sociodemográfico e do questionário Audit. O tratamento estatístico dos dados foi realizado com a utilização da análise bivariada, por meio do teste qui-quadrado ou do teste de Fisher e de modelos multivariados de regressão logística.

Resultados: Observou-se que o álcool é utilizado por $76,6 \%$ dos estudantes pesquisados, e $53,7 \%$ praticavam o BPE. O consumo de bebidas alcoólicas teve associação significativa com a idade e o fato de não morar com a família e estar acima do terceiro ano de graduação. O BPE, por sua vez, esteve associado com o sexo masculino e com o fato de não morar com a família. Conclusões: Este trabalho mostrou que o consumo de álcool entre os estudantes de Medicina é maior do que o da população geral e que a prática do BPE possui alta prevalência nesse grupo. Idade, não morar com a família e período mais avançado do curso foram associados a maior consumo de álcool. Ser do sexo masculino e não morar com a família se associaram a maior risco de BPE.

\section{ABSTRACT}

Objectives: The study had as its goal to verify the pattern of the alcoholic beverages consumption and the presence of drinking heavily snatchy (BPE) among the Medicine students of a Minas Gerais Midwest university. Methods: It is about a transversal, exploratory and descriptive study, performed on 202 graduating students of the Medicine course of a Minas Gerais Midwest university. The data collet was performed on the first term of 2015, a sociodemographic questionnaire and the Audit questionnaire were used. The statistical treatment of the data was performed with the use of the bivariate analysis, through the chi-square test

1 Universidade Federal de São João del-Rei (UFSJ), Divinópolis, MG, Brasil.

Recebido em

20/9/2017

Aprovado em
$25 / 1 / 2018$ 


\section{Keywords}

Alcoholism, Medicine students, alcohol drinking in college, binge drinking, alcohol-related disorders. or by the Fisher test, and multivariate logistic regression models. Results: It was noticed that the alcohol is used by $76,6 \%$ of the students surveyed, being that $53,7 \%$ practiced the drinking heavily episodic. The alcoholic beverages consumption had meaningful association with the age, not living with family and being above the $3^{\text {rd }}$ year of graduation. Drinking heavily episodic had meaningful association with the males and not living with family. Con-

clusions: This paperwork has shown that the alcohol consumption among Medicine students is bigger than among the general population and the binge drink practice has high prevalence on that group. Age, not living with the family and more advanced period of the course were associated with higher alcohol consumption. Being male and not living with the family were associated with a higher risk of BPE.

\section{INTRODUÇÃO}

O consumo de álcool, uma droga lícita, é um comportamento bastante tolerado na sociedade e está presente em diversas manifestações culturais. Segundo a Organização Mundial da Saúde (OMS), 38,3\% das pessoas com 15 anos ou mais consomem bebidas alcoólicas no mundo, em quantidade média de 17,2 litros de álcool puro por pessoa por ano. Nos Estados Unidos da América, tal prevalência é de 61,5\%, e no Brasil 50\% dos adultos (18 anos ou mais) bebem, sendo a cerveja a bebida de preferência nacional ${ }^{1,2}$.

Atualmente, mais do que a frequência de consumo, tem se levado em conta a quantidade de álcool ingerida durante um episódio de bebedeira. É o beber pesado episódico (BPE), também conhecido como binge drink, que se caracteriza pelo consumo além de cinco doses para homens e além de quatro doses para mulheres em único episódio ${ }^{2}$. No mundo, 7,5\% da população bebem em binge, segundo a OMS; no Brasil, dados do "Il Levantamento Nacional de Álcool e Drogas" apontam que 58\% da população brasileira beberam nesse padrão nos últimos 12 meses $^{1,2}$. O levantamento também chama a atenção para o crescimento de 13 pontos percentuais em tal padrão de consumo na população de bebedores, quando comparado ao estudo anterior².

O consumo nocivo dessa substância expõe o usuário a diversas situações de risco. Tais situações incluem desde riscos a curto prazo como acidentes de trânsito - o mais comum e com consequências mais graves² - até consequências a longo prazo, como o desenvolvimento da cirrose e dos cânceres do aparelho gastrointestinal. Dessa forma, o álcool está relacionado a 5,9\% de todas as mortes do mundo'.

Em se tratando do binge drink, além dos riscos já citados, esse padrão associa-se a demais situações de risco como envolvimento em brigas, vandalismo e prática de sexo sem camisinha, além de problemas legais com autoridades e mau desempenho escolar².

Entre as populações estudadas com relação ao uso do álcool, os graduandos de Medicina formam um dos grupos que têm ganhado destaque nesse contexto. Existem características dessa população que podem contribuir para que haja aumento do consumo de álcool, como alta carga horária, alta densidade de informações, cobrança dentro e fora do ambiente acadêmico e independência financeira tardia. Além disso, o graduando de Medicina está intimamente ligado ao tema, pois, em futuro próximo, será o responsável pelo diagnóstico e o tratamento dos transtornos relacionados ao consumo de álcool³.

Ademais, estudos mostram que o conhecimento acerca do uso abusivo de álcool não é bem incorporado pelos estudantes de Medicina, ou eles sofrem forte influência da propaganda e da aceitabilidade social, situações que se confirmam pelo fato de essa droga ser a mais utilizada entre esses estudantes ${ }^{4,5}$. O próprio conhecimento sobre o uso abusivo dessa substância é questionável, segundo a literatura, pois estudos mostram que o ensino sobre o consumo do álcool durante a formação acadêmica na área da saúde é deficiente, visto que, na grade curricular da maioria das instituições, esse tema não é abordado. Esse déficit poderia dificultar a prevenção do uso dessa substância pelo profissional e sua futura conduta perante pacientes que fazem uso de álcool ${ }^{6-9}$.

Para esse grupo, têm sido demonstrados percentuais de consumo de álcool superiores aos da população em geral, podendo ser encontrado índice de 92,8\% na literatura. O BPE e seus danos, como deixar de fazer o que era esperado em decorrência do uso de bebidas alcoólicas, também foram constatados ${ }^{5}$.

Sendo assim, este estudo justifica-se pelo incipiente número de estudos na literatura que abordam o binge drink no cotidiano dos estudantes de Medicina, tendo em vista a relevância e os impactos do tema no contexto social e de formação profissional. Diante disso, esta pesquisa tem como objetivo verificar o padrão de consumo de bebidas alcoólicas e a presença do BPE entre os estudantes de Medicina de uma universidade do centro-oeste de Minas Gerais.

\section{MÉTODOS}

Trata-se de um estudo transversal e descritivo, realizado em uma universidade pública do centro-oeste de Minas Gerais. 
A população eleita foi a de estudantes matriculados no curso de graduação em Medicina, que, no primeiro semestre de 2015, totalizaram 248 alunos, divididos entre o primeiro e o décimo período. Esse número foi considerado para a realização do cálculo amostral, que levou em conta um nível de confiança de $99 \%$ e um erro amostral de 5\%, sendo estimado, portanto, um número de 181 estudantes. Considerando uma perda de 10\%, obteve-se a coleta de dados de 202 acadêmicos, em que foi respeitado o critério de inclusão idade acima de 18 anos. Os estudantes do $11^{\circ}$ e $12^{\circ}$ período foram excluídos da pesquisa em virtude de se encontrarem em internato em campos diversos e em vários municípios, não viabilizando a coleta.

O instrumento utilizado foi um questionário autoaplicável dividido em duas partes. Com o intuito de verificar os dados sociodemográficos, na primeira parte, foram solicitadas informações sobre: sexo, idade, religião, cor da pele, renda familiar, se reside ou não com a família e período em curso. Na segunda parte, foi aplicado o Teste para Identificação de Problemas Relacionados ao Álcool (Audit), versão autoaplicável9. Esse questionário é um instrumento desenvolvido pela OMS com a finalidade de identificar pessoas com consumo de risco, uso nocivo e dependência de álcool, e avalia o consumo dessa substância nos últimos 12 meses. O Audit foi validado no Brasil, em 1999, por Mendez ${ }^{11}$.

As três primeiras questões do questionário estão relacionadas ao padrão de consumo do álcool, como quantidade e frequência de uso da substância. As questões de número 4, 5 e 6 relacionam-se a sinais de dependência e, por fim, as últimas quatro questões analisam o uso prejudicial de álcool. O escore Audit classifica os indivíduos por níveis de risco em Zona I (consumo de baixo risco: 0 a 7 pontos), Zona II (uso de risco: 8 a 15 pontos), Zona III (uso nocivo: 16 a 19 pontos) e Zona IV (provável dependência: 20 a 40), com aumento progressivo dos riscos tanto maiores sejam as pontuações ${ }^{10}$.

O hábito de consumo de bebidas alcoólicas foi identificado por meio da questão 1 do Audit: "Com que frequência você consome bebidas alcoólicas", sendo a resposta "nunca" interpretada como o participante não possuir o hábito de beber; e uma resposta diferente de "nunca", como possuir o hábito de beber. Já o BPE foi definido como qualquer resposta diferente de "nunca" para a questão 3 do Audit: "Qual a frequência com que você consome seis ou mais doses de bebida alcoólica em uma ocasião"12.

A coleta de dados foi realizada no primeiro semestre de 2015. Antes da realização da coleta, os participantes assinaram o Termo de Consentimento Livre e Esclarecido e, após, foi-lhes apresentado o instrumento de coleta de dados.

A análise dos dados foi realizada pelo programa estatístico Statistical Package for the Social Sciences (SPSS), versão 20.0, utilizando-se o teste do qui-quadrado (ou teste exato de Fisher, quando necessário), com nível de significância de 5\%. Foram ajustados modelos de regressão logística multi- variada, considerando-se dois possíveis desfechos: hábito de beber e prática do BPE. Todas as variáveis que apresentaram valores de $\mathrm{P}$ inferiores a 0,20 nas associações bivariadas foram incluídas nas modelagens. No modelo final, após a execução de procedimentos reversos não automáticos, foram mantidas aquelas com valores de $P$ inferiores a 0,05 . Para essas, foram calculadas as razões de chance (odds ratio) e seus respectivos intervalos de confiança. A avaliação do ajuste dos modelos foi realizada por meio de gráficos de resíduos padronizados e pelo teste de Hosmer-Lemeshow.

A pesquisa foi aprovada pelo Comitê de Ética em Pesquisa com Seres Humanos da Universidade Federal de São João del-Rei (Coep) sob o Parecer n 864.946, seguindo as exigências estabelecidas pela Resolução nº 466/2012 do Conselho Nacional de Saúde (CNS).

\section{RESULTADOS}

Do total de alunos regularmente matriculados do primeiro ao décimo período, obtiveram-se 202 participantes, 81\% da amostra total disponível. Um dos participantes, entretanto, foi excluído por não ter respondido ao questionário por completo. Entre os acadêmicos investigados, a maioria era do sexo feminino (54,5\%). A faixa de idade predominante estava entre os 21 e 25 anos (61,7\%), seguido pela faixa etária de 20 anos ou menos (27,4\%). A cor predominante foi a branca $(59,7 \%)$; a religião mais praticada foi a católica $(60,2 \%)$; e a maioria não residia com a família $(75,6 \%)$.

Quanto ao consumo de bebidas alcoólicas, 76,6\% dos estudantes afirmaram fazer o uso dessas bebidas. O consumo de álcool foi maior entre homens, chegando a 83,7\% do total dessa parcela da amostra. As variáveis sexo, idade e morar com a família apresentaram significância estatística quando relacionadas ao hábito de beber na análise bivariada. Por outro lado, a cor da pele, a religião e a renda familiar não mostraram associação estatística com o consumo de bebidas alcoólicas na amostra deste trabalho. Para analisar a relação entre o consumo de álcool e o período cursado, dividiram-se os estudantes do curso em dois grupos. No primeiro foram incluídos os alunos do primeiro e segundo ano, enquanto no segundo, alunos do terceiro, quarto e quinto ano. Com isso, observou-se que havia diferença significativa em relação ao consumo de álcool entre os alunos que estavam mais avançados no curso em comparação àqueles dos estágios iniciais (Tabela 1).

O padrão de consumo em BPE foi identificado em 53,7\% dos participantes e, assim como o hábito de beber, foi mais prevalente no sexo masculino (69,6\%). As variáveis sexo, ser praticante de alguma religião e morar com a família apresentaram um $p$ valor estatisticamente significante na análise bivariada. Não houve associação entre o BPE e idade, cor de pele, renda e período cursado (Tabela 2). 
Tabela 1. Associação entre as características sexo, idade, cor, religião, renda e morar com a família dos acadêmicos de Medicina com o hábito de consumir bebidas alcoólicas - Minas Gerais, 2015

\begin{tabular}{|c|c|c|c|}
\hline \multirow{2}{*}{ Características } & \multicolumn{3}{|c|}{ Consumo de bebidas alcoólicas } \\
\hline & $\operatorname{Sim}(N=154)$ & Não (N = 47) & $P$ \\
\hline Sexo & & & 0,029 \\
\hline Masculino & $77(83,7 \%)$ & $15(16,3 \%)$ & \\
\hline Feminino & $77(70,6 \%)$ & $32(29,4 \%)$ & \\
\hline Idade & & & 0,022 \\
\hline$\leq 20$ & $37(67,3 \%)$ & $18(32,7 \%)$ & \\
\hline 21 a 25 & $103(83,1 \%)$ & $21(16,9 \%)$ & \\
\hline$\geq 26$ & $14(63,6 \%)$ & $8(36,4 \%)$ & \\
\hline Cor de pele & & & 0,484 \\
\hline Branca & $94(78,3 \%)$ & $26(21,7 \%)$ & \\
\hline Outra & $60(74,1 \%)$ & $21(25,9 \%)$ & \\
\hline Praticante de religião & & & 0,189 \\
\hline Sim & $121(74,7 \%)$ & $41(25,3 \%)$ & \\
\hline Não & $33(84,6 \%)$ & $6(15,4 \%)$ & \\
\hline Renda familiar & & & 0,811 \\
\hline 1 salário mínimo & $6(75 \%)$ & $2(25 \%)$ & \\
\hline Entre 2 e 4 & 46 (73\%) & $17(27 \%)$ & \\
\hline Entre 4 e 6 & $41(80,4 \%)$ & $10(19,6 \%)$ & \\
\hline Maior que 6 & $61(77,2 \%)$ & $18(22,8 \%)$ & \\
\hline Mora com a família & & & 0,011 \\
\hline Sim & $31(63,3 \%)$ & $18(36,7 \%)$ & \\
\hline Não & $123(80,9 \%)$ & $29(19,1 \%)$ & \\
\hline Anos cursados & & & 0,006 \\
\hline Até $3^{\circ}$ ano & $87(70,2 \%)$ & $37(29,8 \%)$ & \\
\hline $3^{\circ}$ ao $5^{\circ}$ ano & $67(87 \%)$ & $10(13 \%)$ & \\
\hline
\end{tabular}

No entanto, ao realizar a análise multivariada, com o controle dos fatores de confusão, algumas das variáveis anteriormente associadas com os desfechos analisados perderam a significância estatística. Assim, no modelo multivariado final, o consumo de bebidas alcoólicas foi maior em indivíduos que não moravam com os pais, que estavam acima do terceiro ano de graduação e que tinham menos de 26 anos, e a maior chance de consumir bebidas alcoólicas se deu entre indivíduos na faixa etária de 21 a 25 anos. No que diz respeito à prática do BPE, houve associação significativa desta, no modelo multivariado final, com sexo masculino e com o fato de o indivíduo não morar com a família (Tabela 3).

O resultado da classificação de risco, baseado no questionário Audit, revelou que 73,3\% dos estudantes foram classificados como "consumo de baixo risco" e os demais ficaram enquadrados como "uso de risco", "uso nocivo" das bebidas alcoólicas e "provável dependência".
Tabela 2. Associação entre as características sexo, idade, cor, religião, renda e moradia com familiares dos acadêmicos de Medicina com a prática do BPE - Minas Gerais, 2015

\begin{tabular}{|c|c|c|c|}
\hline \multirow{2}{*}{ Características } & \multicolumn{3}{|c|}{ Praticam o BPE } \\
\hline & $\operatorname{Sim}(N=108)$ & Não (N = 93) & $P$ \\
\hline Sexo & & & $<0,001$ \\
\hline Masculino & $64(69,6 \%)$ & $28(30,4 \%)$ & \\
\hline Feminino & $44(40,4 \%)$ & $65(59,6 \%)$ & \\
\hline Idade & & & 0,885 \\
\hline$\leq 20$ & $28(50,9 \%)$ & $27(49,1 \%)$ & \\
\hline 21 a 25 & $68(54,8 \%)$ & $56(45,2 \%)$ & \\
\hline$\geq 26$ & $12(54,5 \%)$ & $10(45,5 \%)$ & \\
\hline Cor de pele & & & 0,467 \\
\hline Branca & $67(55,8 \%)$ & $53(44,2 \%)$ & \\
\hline Outra & $41(50,6 \%)$ & $40(49,4 \%)$ & \\
\hline Praticante de religião & & & 0,031 \\
\hline Sim & $81(50 \%)$ & $81(50 \%)$ & \\
\hline Não & $27(69,2 \%)$ & $12(30,8 \%)$ & \\
\hline Renda familiar & & & 0,753 \\
\hline 1 salário & $4(50 \%)$ & $4(50 \%)$ & \\
\hline Entre 2 e 4 & $33(52,4 \%)$ & $30(47,6 \%)$ & \\
\hline Entre 4 e 6 & $25(49 \%)$ & $26(51 \%)$ & \\
\hline Maior que 6 & $46(58,2 \%$ & $33(41,8 \%)$ & \\
\hline Mora com a família & & & 0,016 \\
\hline Sim & $19(38,8 \%)$ & $30(61,2 \%)$ & \\
\hline Não & $89(58,6 \%)$ & $63(41,4 \%)$ & \\
\hline Anos cursados & & & 0,445 \\
\hline Até $3^{\circ}$ ano & $64(51,6 \%)$ & $60(48,4 \%)$ & \\
\hline $3^{\circ}$ ao $5^{\circ}$ ano & $44(57,1 \%)$ & $33(42,9 \%)$ & \\
\hline
\end{tabular}

Tabela 3. Variáveis associadas significativamente com o consumo de bebidas alcoólicas e com a prática do BPE - Minas Gerais, 2015: Análise multivariada por meio de modelos de regressão logística

\begin{tabular}{lcc}
\hline \multicolumn{3}{c}{ Desfecho 1:Consumo de bebidas alcoólicas } \\
\hline Exposiçöes & Odds ratio & IC (95\%) \\
\hline Idade: $\leq 20^{*}$ & 1,75 & $0,53-5,75$ \\
Idade: 21 a $25^{*}$ & 3,17 & $1,11-9,01$ \\
Não morar com a família & 2,75 & $1,29-5,84$ \\
$3^{0}$ a0 $5^{\circ}$ ano da faculdade & 2,73 & $1,12-6,65$ \\
\hline & Desfecho 2: Prática do BPE \\
\hline Sexo masculino & 3,30 & $1,82-5,98$ \\
Não morar com a família & 2,13 & $1,07-4,24$ \\
\hline
\end{tabular}

* Categoria de referência $=$ idade $\geq 26$ anos $(O R=1,00)$. 


\section{DISCUSSÃO}

Corroborando os resultados deste estudo, a literatura aponta que a prevalência de consumo de bebidas alcoólicas entre estudantes na área de saúde varia de 64,2\% a 91\%, índice esse maior que o da população brasileira em geral, que é de 50\%. Esses achados indicam maior risco de dependência entre os estudantes, o que pode trazer graves consequências laborais e sociais, uma vez que podem interferir na habilidade de prestar cuidado adequado aos pacientes, quando forem, em breve, profissionais da saúde $\mathrm{e}^{2,45,13-17}$.

O ingresso na academia é um período de transformações importantes na vida do estudante, momento no qual se tem maior acesso ao consumo de álcool e, consequentemente, maior ocorrência de binge drink $k^{16,18}$. Quanto a essa prática, os resultados deste estudo coincidem com os índices da população brasileira em geral, porém apresentam valores maiores quando comparados a outros estudos realizados entre estudantes de Medicina, fato que reflete a individualidade de cada instituição e de seus estudantes 2,5,13,19.

Quando se analisa a diferença de consumo de álcool entre os gêneros, apesar da análise bivariada mostrar diferença significativa entre os sexos quanto ao consumo e à prática do BPE, a análise multivariada concluiu que há predomínio apenas do binge drink pelo sexo masculino, resultado que segue tanto as tendências da população brasileira adulta, de modo geral, quanto os resultados das pesquisas com estudantes $24,5,14,16,20,21$. Entre as possíveis explicações para a maior prática do BPE por homens, a possibilidade da melhora no desempenho sexual com o uso do álcool tem sido apontada como justificativa para a população masculina em âmbito geral21,22.

O quesito "morar com a família" apresenta-se como possível fator não associado tanto para o consumo de bebidas alcoólicas como para a prática do BPE, concordando com outros resultados encontrados na literatura ${ }^{5,13,14,23,24}$. Com o ingresso na universidade, muitos estudantes deixam suas famílias e passam a morar com outros estudantes, o que favorece $\mathrm{o}$ acesso às bebidas alcoólicas ${ }^{25}$. Além disso, o sentimento de liberdade proporcionado por não morar com os pais é também citado pela literatura como fator desencadeante do uso excessivo de bebidas alcoólicas ${ }^{26}$.

Quanto à prática religiosa e o consumo de álcool, o resultado na amostra desta pesquisa vai na contramão da maioria dos estudos encontrados, já que a análise multivariada não demonstrou associação entre praticar uma religião e o consumo de álcool. Estudos encontrados na literatura demonstram que, em suas amostras, a religião está associada negativamente tanto com o consumo de álcool quanto com a prática do BPE ${ }^{5,13,16,27}$, o que pode ser justificado pelo fato de as religiões modularem, muitas vezes, o consumo dessa substância. Desse modo, pertencer a um grupo com valores estabelecidos e normas compartilhadas que condenam o uso dessa substância pode favorecer a sua recusa ${ }^{28}$.
O consumo de bebidas alcoólicas em relação ao período cursado varia conforme os estudos, algumas vezes concordando com os achados deste trabalho ao mostrar maior prevalência nos períodos mais avançados ${ }^{23}$, outras vezes, não mostrando diferença estatística entre essas variáveis ${ }^{15,19}$. A associação entre o maior consumo de bebidas alcoólicas pelos alunos de períodos mais avançados pode ser justificada pelo fato de que, à medida que caminham no curso, esses estudantes, possivelmente detentores de maior carga de conhecimento acerca do tema, desenvolvem a convicção de que serão capazes de controlar os problemas que eventualmente possam surgir a partir do uso indevido do álcool e outras drogas ${ }^{29}$. Esse dado torna-se de grande importância, visto que esses estudantes poderão subestimar o diagnóstico e o tratamento do alcoolismo de seus futuros pacientes ${ }^{3,30}$.

Já em relação ao BPE, corroborando este estudo, uma pesquisa realizada com estudantes de uma universidade de Montes Claros, MG, em 2015, também não obteve associação estatística significativa entre a prática de binge drink e o período cursado pelo estudante ${ }^{16}$. A literatura nos demonstra, ainda, que há uma tendência à redução da prática de binge drink com o aumento da escolaridade, segundo um levantamento realizado pelo Ministério da Saúde ${ }^{31}$.

Ao avaliar os estudantes por faixa etária, observou-se predomínio do consumo de álcool entre aqueles que tinham menos de 26 anos, com maior prevalência entre 21 e 25 anos. Tal achado pode refletir uma tendência à diminuição do uso da substância com o avançar da idade, o que pode ser, de certa forma, demonstrado pelo achado do Ministério da Saúde de diminuição do consumo abusivo de bebidas alcoólicas a partir dos 35 anos $^{31}$. Já a prática do BPE não está associada com a idade no nosso estudo.

Ao aplicar o Audit, foi identificado que 96,5\% dos estudantes se encontravam nas zonas de consumo de baixo risco e consumo de risco. Para esses estudantes, orientações quanto aos perigos do consumo abusivo dessa substância deviam ser realizadas, a fim de evitar a progressão para as zonas de maior risco ${ }^{32}$.

A prevalência de estudantes que se encontravam em uso nocivo e provável dependência (3,5\%) concordou com os achados de outros estudos realizados sobre a temática, que mostravam prevalências semelhantes ou até mesmo superiores do uso nocivo do álcool 19,23,33. Esses estudantes que se encontram em uso nocivo e provável dependência requeriam avaliação, monitoração contínua e tratamento, uma vez que o uso abusivo traz graves consequências às atividades laborais, como prejuízo na habilidade de aprendizado, na memória, na velocidade psicomotora e na tomada de decisões, fatores cruciais ao exercício futuro da profissão ${ }^{6}$.

A principal limitação deste estudo está no fato de o BPE não ter sido avaliado por um instrumento próprio, mas, sim, pelo Audit, cujo objetivo primário é avaliar o risco de consumo de álcool, e não o padrão em binge drink, podendo, 
assim, gerar algum viés. Além disso, o fato de não incluir os alunos do $11^{\circ}$ e $12^{\circ}$ período, reduziu o tamanho da amostra, não permitindo avaliar o consumo do álcool e o BPE durante o último ano do curso, o que poderia reforçar ou refutar o aumento do consumo de álcool com o avançar da graduação.

\section{CONCLUSÕES}

O presente trabalho mostra que o consumo de álcool entre os estudantes de Medicina é maior do que o da população geral e que a prática do BPE foi identificada em 53,7\% dos participantes, evidenciando-se a alta prevalência de tais práticas nesse grupo. A partir da análise multivariada, observou-se que a idade entre 21 e 25 anos, estar entre o terceiro e quinto ano do curso e o fato de não morar com a família associaram-se com o hábito de consumir bebidas alcoólicas. Em relação ao beber em binge drink, os fatores associados foram ser do sexo masculino e não morar com a família.

Espera-se que o presente trabalho sirva de alerta às autoridades de saúde para que haja uma abordagem efetiva acerca do consumo de álcool e do BPE durante a graduação de Medicina, bem como sirva de apoio para pesquisas futuras.

\section{CONTRIBUIÇÕES INDIVIDUAIS}

Alexandre Ernesto Silva - Realizou revisão bibliográfica, coleta de dados, preenchimento do banco de dados, análise e interpretação dos resultados, e escrita do texto.

Amanda de Oliveira Maurílio - Realizou revisão bibliográfica, coleta de dados, preenchimento do banco de dados, análise e interpretação dos resultados, e escrita do texto.

Caio César Liguori - Realizou revisão bibliográfica, COleta de dados, preenchimento do banco de dados, análise e interpretação dos resultados, e escrita do texto.

Daniel Vasconcelos de Pinho Tavares - Realizou revisão bibliográfica, coleta de dados, preenchimento do banco de dados, análise e interpretação dos resultados, e escrita do texto.

Delmo Manoel Gomes Terceiro - Realizou revisão bibliográfica, coleta de dados, preenchimento do banco de dados, análise e interpretação dos resultados, e escrita do texto.

Lucas Geraldo Malta Cunha - Realizou revisão bibliográfica, coleta de dados, preenchimento do banco de dados, análise e interpretação dos resultados, e escrita do texto.

Thalles Trindade de Abreu - Realizou revisão bibliográfica, coleta de dados, preenchimento do banco de dados, análise e interpretação dos resultados, e escrita do texto.

Vinicius Silva Belo - Realizou análise e interpretação dos resultados, e escrita do texto.

\section{CONFLITO DE INTERESSES}

Não existe.

\section{REFERÊNCIAS}

1. World Health Organization. Global status report on alcohol and health 2014. Disponível em: http://www.who.int/substance_abuse/publications/global_alcohol_report/en/. Acesso em: 5 jun. 2014.

2. II Levantamento Nacional de Álcool e Drogas (Lenad) - 2012. Ronaldo Laranjeira, et al. supervisão. São Paulo: Instituto Nacional de Ciência e Tecnologia para Políitcas Públicas de Álcool e Outras Drogas (Inpad), Unifesp; 2014.

3. Gonçalves SS, Silvany Neto AM. Dimensão psicológica da qualidade de vida de estudantes de medicina. Rev Bras Educ Med. 2013;37(3):385-95.

4. Machado JNS, Finelli LAC, Jones KM, Soares WD. Consumo de álcool entre acadêmicos de medicina. Rev Bras Pesq Ciênc Saúde. 2015;2(2):46-51.

5. Carneiro EB, Braga RT, Silva LF, Nogueira MC. Fatores associados a beber pesado episódico entre estudantes de medicina. Rev Bras Educ Med. 2012;36(4):524-30.

6. Mesquita EM, Nunes AJ, Cohen C. Avaliação das atitudes dos estudantes de medicina frente ao abuso de drogas por colegas do meio acadêmico. Rev Psiquiatr Clín. 2008;35(Supl 1):8-12.

7. Santos VOG. A construção do conhecimento acerca do fenômeno das drogas: desafios no ensino crítico e problematizador [dissertação]. Rio de Janeiro: Universidade do Estado do Rio de Janeiro; 2010

8. Amorim AVC, Kikko EO, Abrantes MM, Andrade VLA. Álcool e alcoolismo: estudo de prevalência entre discentes do curso de medicina da Unifenas em Belo Horizonte - Minas Gerais. Rev Med Minas Gerais. 2008;18(1):16-23.

9. Lemos KM, Neves NMBC, Kuwano AY, Tedesqui G, Bitencourt AGV, Neves FBCS, et al. Uso de substâncias psicoativas entre estudantes de Medicina de Salvador (BA). Rev Psiquiatr Clín. 2007;34(3):118-24

10. Saunders JB, Aasland OG, Babor TF, de la Fuente JR, Grant M. Development of the Alcohol Use Disorders Identification Test (AUDIT): WHO Collaborative Project on Early Detection of Persons with Harmful Alcohol Consumption - II. Addiction. 1993;88(6):791-804.

11. Mendez EB. Uma versão brasileira do Audit - Alcohol Use Disorders Identification Test [Dissertação]. Pelotas: Universidade Federal de Pelotas; 1999.

12. Shakeshaft AP, Bowman JA, Sanson-Fisher RW. Comparison of three methods to assess binge consumption: one-week retrospective drinking diary, AUDIT, and quantity/frequency. Subst Abus. 1998;19(4):191-203.

13. Carneiro ALM, Rodrigues SB, Gherardi-Donato ECS, Guimarães EAA, Oliveira VC. Padrão do uso de álcool entre estudantes universitários da área de saúde. Rev Enferm Cent 0 Min. 2014;4(1):940-50.

14. Ramis TR, Mielke Gl, Habeyche EC, Oliz MM, Azevedo MR, Hallal PC. Tabagismo e consumo de álcool em estudantes universitários: prevalência e fatores associados. Rev Bras Epidemiol. 2012;15(2):376-85.

15. Petroianu A, Reis DCF, Cunha BDS, Souza DM. Prevalência do consumo de álcool, tabaco e entorpecentes por estudantes de Medicina da Universidade Federal de Minas Gerais. Rev Assoc Med Bras. 2010;56(5):568-71.

16. Cardoso FM, Barbosa HA, Costa FM, Vieira MA, Caldeira AP. Fatores associados à prática do binge drinking entre estudantes da área da saúde. Rev CEFAC. 2015;17(2):475-84.

17. Silva VA, Aguiar AS, Felix F, Rebello GP, Andrade RC, Mattos HF. Estudo brasileiro sobre abuso de substâncias por adolescentes: fatores associados e adesão ao tratamento. Rev Bras Psiquiatr. 2003;25(3):133-8.

18. Wagner GA, Andrade AG. Uso de álcool, tabaco e outras drogas entre estudantes universitários brasileiros. Rev Psiquiatr Clin. 2008;35(Supl1):48-54.

19. Rocha LA, Lopes ACFMM, Martelli DRB, Lima VB, Martelli-Júnior H. Consumo de álcool entre estudantes de faculdades de Medicina de Minas Gerais, Brasil. Rev Bras Educ Med. 2011;35(3):369-75

20. Moura EC, Malta DC. Consumo de bebidas alcoólicas na população adulta brasileira: características sociodemográficas e tendência. Rev Bras Epidemiol. 2011;14(Supl 1):61-70. 
21. Fachini A, Furtado EF. Uso de álcool e expectativas do beber entre universitários: uma análise das diferenças entre os sexos. Psic Teor Pesq. 2013;29(4):421-8.

22. Holmila M, Raitasalo K. Gender differences in drinking: why do they still exist? Addiction. 2005;100(12):1763-9.

23. Barbosa FL, Barbosa RL, Barbosa MCL, Aguiar DL, Figueiredo IA, Ribeiro AC, et al. Uso de álcool entre estudantes de medicina da Universidade Federal do Maranhão. Rev Bras Educ Med. 2013;37(1):89-95.

24. Rodrigues PFS, Salvador ACF, Lourenço IC, Santos LR. Padrões de consumo de álcool em estudantes da Universidade de Aveiro: relação com comportamentos de risco e stress. Aná Psicologica. 2014;32(4):453-66.

25. Zalaf MRR, Fonseca RMGS. Uso problemático de álcool e outras drogas em moradia estudantil: conhecer para enfrentar. Rev Esc Enferm. USP. 2009;43(1):132-8.

26. Windle M. Alcohol use among adolescents and young adults. Population. 2003;45(5.9):15-9.

27. Silva LVER, Malbergier A, Stempliuk VA, Andrade AG. Fatores associados ao consumo de álcool e drogas entre estudantes universitários. Rev Saúde Pública 2006;40(2):280-8.

28. Pillon SC, Santos MA, Gonçalves AMS, Araújo KM, Funai A. Fatores de risco, níveis de espiritualidade e uso de álcool em estudantes de dois cursos de enfermagem. SMAD Rev
Eletrônica Saúde Mental Álcool Drog. 2010;6(1):493-513. Disponível em: http://pepsic. bvsalud.org/scielo.php?script=sci_arttext\&pid=\$1806-69762010000300008. Acesso em: 28 maio 2015.

29. Nóbrega MPSS, Simich L, Strike C, Brands B, Giesbrecht N, Khenti A. Policonsumo simultâneo de drogas entre estudantes de graduação da área de ciências da saúde de uma universidade: implicações de gênero, sociais e legais, Santo André - Brasil. Texto Contexto Enferm. 2012;21(spe):25-33.

30. Santos MVF, Pereira DS, Siqueira MM. Uso de álcool e tabaco entre estudantes de Psicologia da Universidade Federal do Espírito Santo. J Bras Psiquiatr. 2013;62(1):22-30.

31. Brasil. Ministério da Saúde. Vigitel Brasil 2016: vigilância de fatores de risco e proteção para doenças crônicas por inquérito telefônico: estimativas sobre frequência e distribuição sociodemográfica de fatores de risco e proteção para doenças crônicas nas capitais dos 26 estados brasileiros e no Distrito Federal em 2016. Brasília: Ministério da Saúde; 2017.

32. Babor TF. Audit - The Alcohol Use Disorders Identification Test - Guidelines for Use in Primary Care. 2nd ed. Geneva: World Health Organization; 2001.

33. Peuker AC. Expectativas e beber problemático entre universitários. Psic Teor Pesq. 2006;22(2):193-200. 